\title{
Orbital Floor Fracture
}

\author{
Hyo Seong Kim ${ }^{1,2}$, \\ Eui Cheol Jeong ${ }^{1,2}$ \\ ${ }^{1}$ Department of Plastic Surgery, SMG-SNU \\ Boramae Medical Center, Seoul; \\ ${ }^{2}$ Department of Plastic and Reconstructive \\ Surgery, College of Medicine, Seoul National \\ University, Seoul, Korea
}

No potential conflict of interest relevant to this article was reported.
The medial wall and floor of the bony orbit are frequently fractured because of the delicate anatomy. To optimize functional and aesthetic results, reconstructive surgeons should understand the anatomy and pathophysiology of orbital fractures. Appropriate treatment involves optimal timing of intervention, proper indications for operative repair, incision and dissection, release of herniated tissue, implant material and placement, and wound closure. The following review will discuss the management of orbital floor fractures, with the operative method preferred by the author. Special considerations in operation technique and the complication are also present in this article.

Keywords: Orbital fractures / Blow-out fractures / Surgery / Complications

\section{INTRODUCTION}

Fractures of the orbit are common yet challenging to manage. Isolated orbital fractures are encountered in 4\%-to- $16 \%$ of all facial fractures, and orbital fractures compose $30 \%-55 \%$ of zygomatic complex and naso-orbital-ethmoid fractures [1,2]. Seven facial bones make up the bony orbit: the frontal, maxilla, zygoma, ethmoid, lacrimal, greater and lesser wings of the sphenoid, and palatine bones. The ethmoid, frontal, and maxillary bonesclosely related to sinus pneumatization-act as shock absorbers for the eye globe. The outer rim of the orbit is comprised of the first three robust bony elements, protecting the more delicate internal bones of the orbital cavity. The orbital cavity is itself bound by the orbital roof, lateral and medial walls, and orbital floor. The orbital floor, which forms the roof of the maxillary sinus, slopes upward toward the apex of the pyramid, which lies roughly 44 to $50 \mathrm{~mm}$ posterior to the orbital entrance $[3,4]$. This complicated anatomy makes repair and reconstruction of orbital fracture difficult for a novice (Fig. 1).

Correspondence: Eui Cheol Jeong

Department of Plastic Surgery, SMG-SNU Boramae Medical Center, 20

Boramae-ro 5 gil, Dongjak-gu, Seoul 07061, Korea

E-mail: ejeong.md@gmail.com

Received May 4, 2016 / Revised August 30, 2016 / Accepted August 30, 2016

\section{ANATOMY AND PATHOPHYSIOLOGY}

Of the bony orbit, the thin portion is defined posterolaterally by sphenomaxillary fissure but without any distinct border medially. Most of the thin portion ( $<0.5 \mathrm{~mm}$ in thickness) is medial to the infraorbital groove and canal, which is the roof of the maxillary sinus and the floor of the orbit. The lateral part of the ethmoid sinus, named the lamina papyracea, is the medial wall of the orbit and is also thin [3]. Any or all of the related bones can be involved in orbital fracture. However, the floor or medial wall is more frequently fractured because of the aforementioned anatomical characteristics (Fig. 2).

Orbital floor fractures may occur in isolation ("blowout" fractures) or as part of a zygomaticomaxillary complex fracture. The terms pure and impure have been used to describe isolated orbital fractures (pure) versus orbital fractures that occur in conjunction with other fractures (impure) [5]. Both "buckling" theory and "hydraulic" theory explain the force transmission to the relatively weak orbital floor, resulting in fractures [6].

\section{SURGICAL INDICATION}

Indications for surgical treatment are divided into functional or aesthetic reasons. Functional problems are caused by either im- 


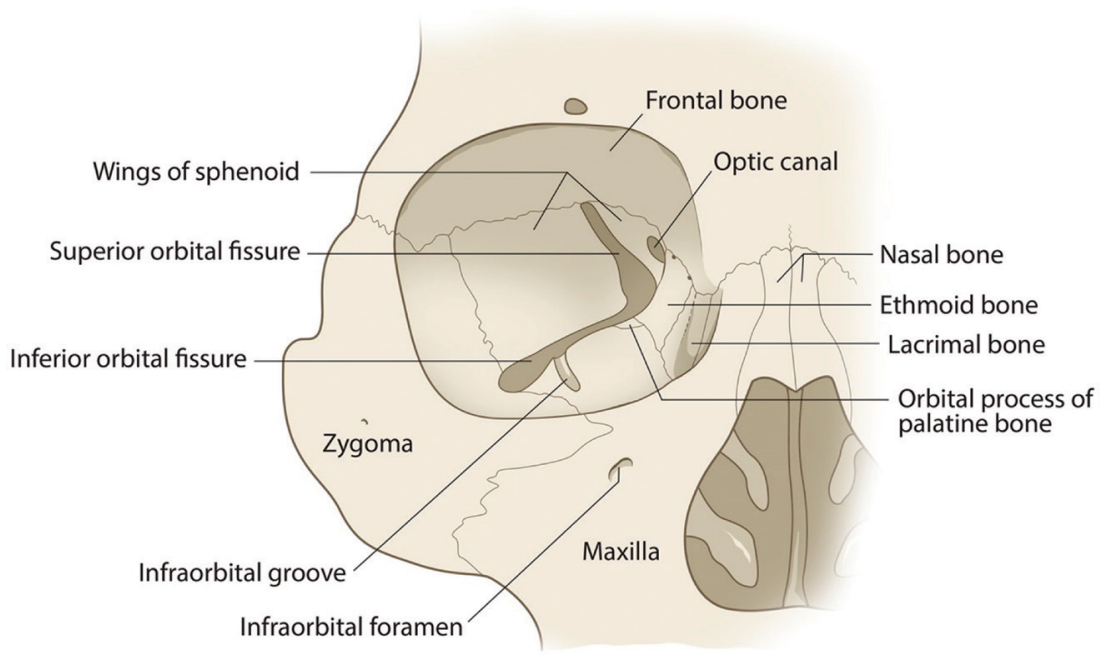

Fig. 1. The orbital wall consists of frontal, ethmoid, lacrimal, maxilla, zygoma, sphenoid, and palatine bones.

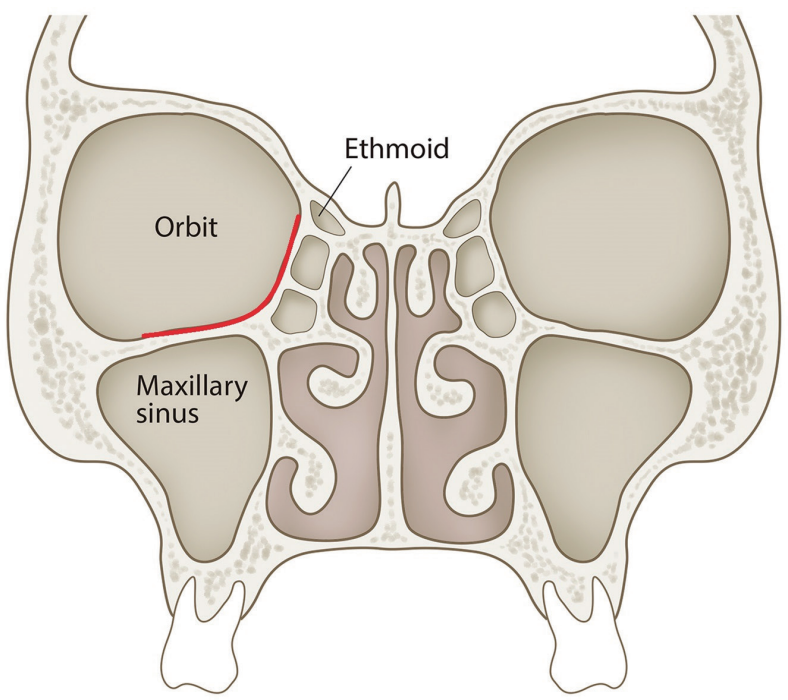

Fig. 2. Common fracture sites of blowout fracture of orbit (red line). Most thin portions of the orbit is medial to the infraorbital groove.

pingement of the soft tissue structure upon or by the fracture, including the periorbital and extraocular muscles (EOM), the motor nerves to the eye globe, vessels, and the trigeminal nerves and the optic nerve, and are rarely related to orbital apex syndrome or superior orbital fissure syndrome [7-9]. Most functional issues are related to entrapment of the inferior rectus muscle or adjacent periorbital soft tissue, which may restrict EOM movement and result in diplopia and sometimes oculocardiac reflex, such as nausea, vomiting, and arrhythmia. Such functional problems can be examined by forced duction test of the affected muscle (Fig. 3).

In the pediatric population, the bony elasticity allows the fracture to open and close back into position, tightly trapping the periorbital tissue or EOMs. This clinical presentation is referred as the white-eyed blowout fracture (age $<18$ years), which involves a history of periorbital trauma, little ecchymosis or edema (lack of black eye), but marked EOM restriction [10,11].

With white-eyed blowout fracture, ischemia can cause permanent damage to the involved EOM with resultant Volkmann contracture of extraocular muscles (VC-EOM). Because of this, 

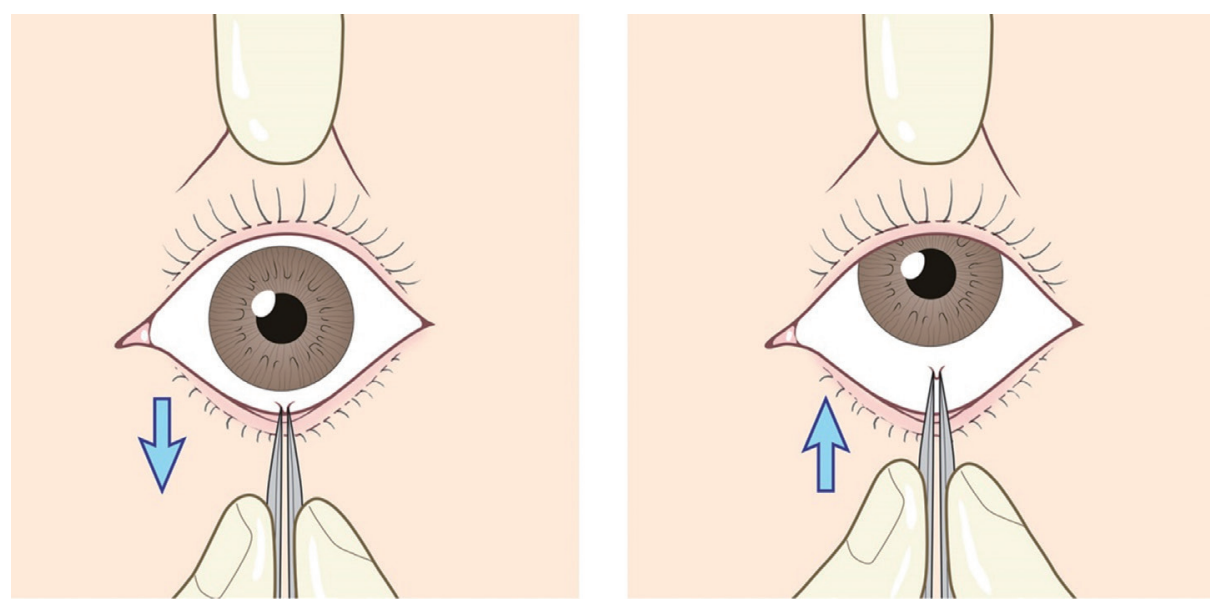

Fig. 3. Forced duction test should be performed to evaluate extraocular muscle entrapment. The examiner uses forceps to grasp the conjunctiva near the attachment of the inferior rectus muscle and attempts to move the globe through a full range of motion. Because of potential significant discomfort, this should be performed under sedation or anesthesia.

white-eyed blowout fracture is considered a surgical emergency and warrants operative intervention within 24 hours of injury. In addition, VC-EOM could also occur in orbital compartment syndrome, in elderly patients who are hypotensive, and in those with small-fracture diplopia [12].

Aesthetic indications for surgical interventions involve globe malposition. Enophthalmos or hypoglobusis are commonly encountered. The severity of these conditions dictates the need for an observation period of about 2 weeks. In recent literature, early surgical repair has been recommended because it was associated with better outcomes $[13,14]$. Most surgeons agree that a $2 \times 2-\mathrm{cm}$ defect or defects larger than $50 \%$ of the wall behind the equator of the globe is most likely to cause clinically significant enophthalmos (greater than $2 \mathrm{~mm}$ ), and these patients are candidates for surgical repair [15].

Relative contraindication are reserved for ocular injuries, such as hyphema, globe perforation, and retinal tears. Additional relative contraindications include patients with vision only in the eye in the affected orbit, those who have undergone recent ophthalmologic surgery, and those with medical instability [16]. Ophthalmologic evaluation and clearance are necessary in such cases prior to any manipulation of the bony orbit or periorbital soft tissue (Table 1).
Table 1. Indication and contraindication of surgical repair

Surgical indications of orbital floor fractures are in the following cases: - Mechanical restriction of gaze with a positive forced duction test of computed tomography (CT) evidence of incarcerated EOM - Larger defect that is greater than half the surface in CT scan or with prolapsed orbital soft tissue

- Clinical enophthalmos (>2 mm) or hypophthalmos with serial examinations in following 2 weeks

Contraindications to surgery are in the following cases:
- Hyphema
- Retinal tears
- Globe perforation
- Only-seeing eye
- Medical instability

EOM, extraocular muscles.

\section{SURGICAL APPROACH}

Traditionally, the orbital floor is accessed transcutaneously via infraorbital or lower eyelid incision or via the transconjunctival approach (Fig. 4). The type of surgical access is chosen in consideration of the location and size of the fracture and of the surgeon's preference and experience. An alternate option is the endoscopic approach. This involves minimal globe manipulation, and sometimes, may be applicable in patients for whom a surgical intervention is contraindicated via traditional access. As with any endoscopic approach, it is important to discuss with the patient the possibility that an additional traditional approach may have to be 


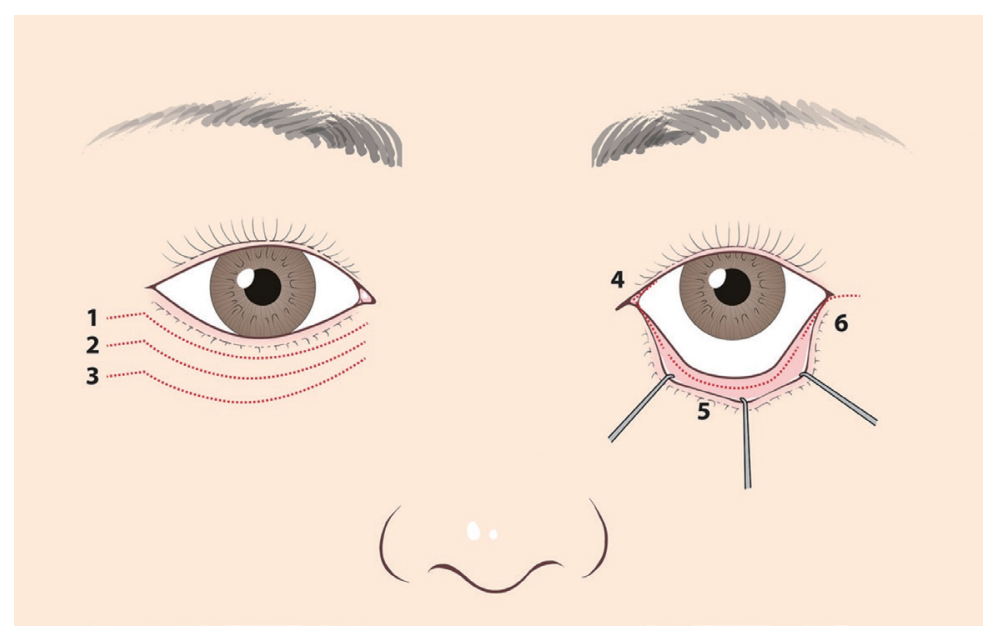

Fig. 4. Types of accesses used to expose the orbital floor: (1) subciliary, (2) subtarsal, (3) infraorbital, (4) transcaruncular, (5) tranconjuctival, and (6) transconjunctival with lateral canthotomy.

used if endoscopic repair fails [17].

The author's preferred method for accessing the orbital floor is via the transconjunctival approach (Fig. 5). It provides adequate visualization of the orbital floor. If further exposure is required, the access can be extended with a lateral canthotomy. A subciliary or midlid incision also allows exposure of the orbital floor but requires an external cutaneous incision. Although the latter options may provide superior exposure, the incisions carry a higher risk of visible scars and subsequent ectropion [18].

With the needle electrocautery on a lower power setting, an incision is made from the medial to lateral or lateral to medial side through the conjunctiva, $1 \mathrm{~mm}$ below the inferior margin of the tarsal plate. A preseptal dissection is made down toward the inferior orbital rim because this creates for a more hemostatic field without orbital fat herniation and a lower risk of retrobulbar hematoma. After the infraorbital rim has been exposed, subperiosteal dissection is carried out posteriorly. The dissection can be safely extended $25 \mathrm{~mm}$ posteriorly from the inferior and lateral rim. The infraorbital nerves, the origin of the inferior oblique muscle, lacrimal apparatus, and optic nerves are special structures for which disruption should be avoided during operation. The author prefers to dissect from the lateral to medial side. This makes identification of the important anatomic structures and reduction of herniated fat tissue from the fracture site easy and safe (Fig. 6). Care must be taken to avoid aggressive traction of the soft tissue because this may cause further bleeding deep in the orbit. Intraoperative bleeding can obscure the surgical field, which can be easily controlled by identification of the source and the use of bipolar or low-power electrocautery. The endpoint of dissection is reached when the surgeon has reduced all of the herniated tissue in the orbit and has exposed the bone of the unfractured orbit in a circumferential fashion. Even in a large fracture, the posterior ledge of the unfractured orbit can safely be identified by placing the elevator in the maxillary sinus and sweeping it upward and forward (Fig. 5). The unfractured posterior ledge is usually the palatine bone.

Once the size and shape of the defect have been assessed, an autogenous graft or alloplastic implant is placed above the orbital shelf. The author's preference is to use a thin sheet of porous polyethylene or titanium mesh that is secured with one or two screws just behind the anterior rim of the orbit. In children under 8 years of age, autogenous bone graft or absorbable material should be used to accommodate growth of the orbital skeleton.

A forced duction test is repeated after the implant has been placed to ensure eye mobility. The wound is inspected for hemostasis and then copiously irrigated. To prevent ectropion by septal shortening, the released periosteum (arcus marginalis) is reattached to the orbit rim, not to the anterior lamella of the orbicularis oculi muscle. 

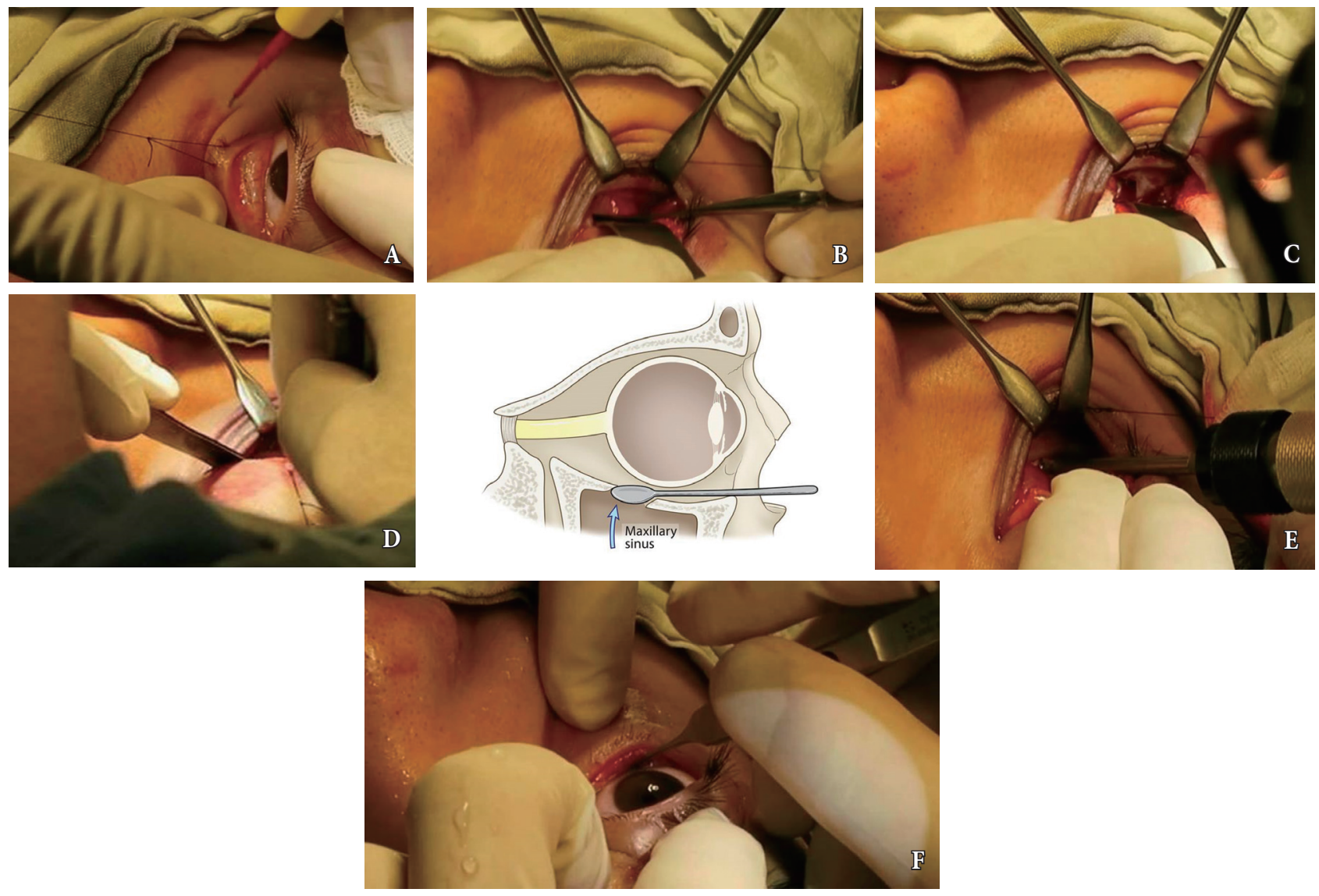

Fig. 5. Transconjunctival access to orbital floor (author's preferred technique). (A) Transconjunctival incision $1 \mathrm{~mm}$ below inferior margin of tarsal plate. (B) After the preseptal dissection and release of arcus marginalis, subperiosteal dissection is carried out posteriorly. (C) A periosteal elevator in combination with a small malleable retractor is used to identify the margins of the fracture to free an impinged or herniated tissue. Gentle manipulation is essential to avoid intraoperative bleeding. (D) Identification of posterior shelf. The elevator is placed in the maxillary sinus and swept upward to reveal the posterior shelf. (E) Implant is usually secured with a single screw. (F) The forced duction test is repeated after implant placement.

\section{ACUTE COMPLICATIONS}

There are numerous acute complications from orbital fracture repair, which can include permanent loss of vision in the affected eye. Intraoperative bleeding, excessive dissection of the orbit posteriorly, or deep insertion of the implant can cause impingement on the orbital apex or retrobulbar hematoma, possibly resulting in vision loss. Gentle tissue handing and visual confirmation of the implant placement above the remaining orbital shelf and below the periorbital tissue are necessary to avoid these serious complications. Any clinical sign of postoperative retrobulbar hematoma should be addressed immediately by lateral canthotomy, medical treatment to lower intraocular pressure, and surgical exploration of the orbit [19].

\section{LATE COMPLICATIONS AND REVISION SURGERY}

The most common late complications following surgical repair of the orbital floor are ectropion, persistent postoperative diplopia, infraorbital nerve dysfunction, and enophthalmos. Implant-associated infections include implant migration, expo- 


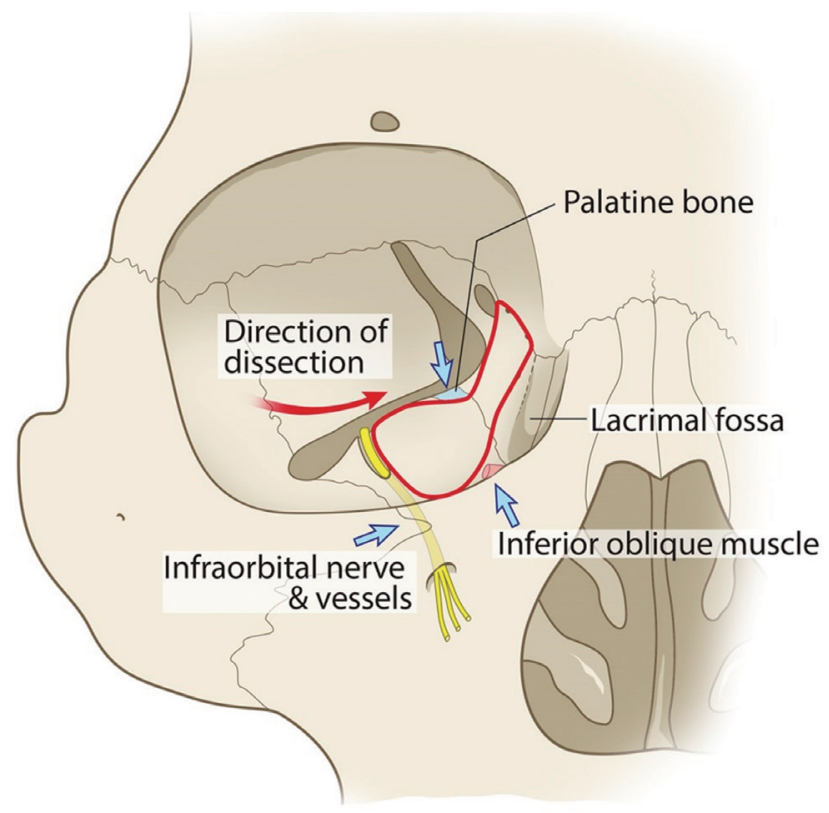

Fig. 6. Subperiosteal dissection in the posterior area usually starts from lateral to medial side (red arrow) to allow for ready identification of the important structures (blue arrows): infraorbital nerves and vessels, origin of inferior oblique muscle, and orbital process of palatine bone, which is usually the posterior shelf.

sure, palpability, or local inflammatory reaction, and serious complications include postoperative optic neuropathy, blindness, and retrobulbar hematoma, but these are fortunately very rare [19-21].

Secondary orbital reconstruction should be considered in the case of prolonged diplopia, enophthalmos and hypophthalmos, telecanthus, contour abnormality, or eyelid deformity [19,21]. However, reoperation is technically demanding because of the extensive dissection required. If necessary, secondary reconstructions should be delayed for at least 6 months after the primary operation to allow for adequate healing and neuromuscular recovery.

Persistent diplopia in the postoperative setting can be a result of EOM deficits. A normal forced duction test should effectively rule this out. Frequently, periorbital swelling or muscle contusion may be the underlying cause, as the majority of these cases are resolved within 8 weeks. If forced duction test reveals entrapped soft tissue, a computed tomography study is needed to determine if the implant is causing interference with the EOMs. If so, reoperation should be considered (Fig. 7).
Postoperative enophthalmos is also a common problem in orbital fracture management. The majority of cases are secondary to enlargement of the bony orbit because of slight imperfections in reconstructing the orbital anatomy. In some cases, the existing implant should be elevated or repositioned. Additional volume should be added to the orbit in a posterolateral location if the implant insertion does not result in an appropriated globe position. There are various implants available for orbital floor reconstruction. The author prefers to use preformed orbital reconstruction plates for secondary reconstruction of enophthalmos (Fig. 8) [22-24].

Ancillary soft tissue operation of the eyelid should be conducted in selective cases for the enhancement of aesthetic condition. Cosmetic eyelid surgery can improve the symmetry of the supratarsal crease or vertical height of the palpebral fissure in mild cases of postoperative enophthalmia. Operative ectropion or entropion improves after 4 to 6 months of conservative therapy, but sometimes requires surgical intervention. 

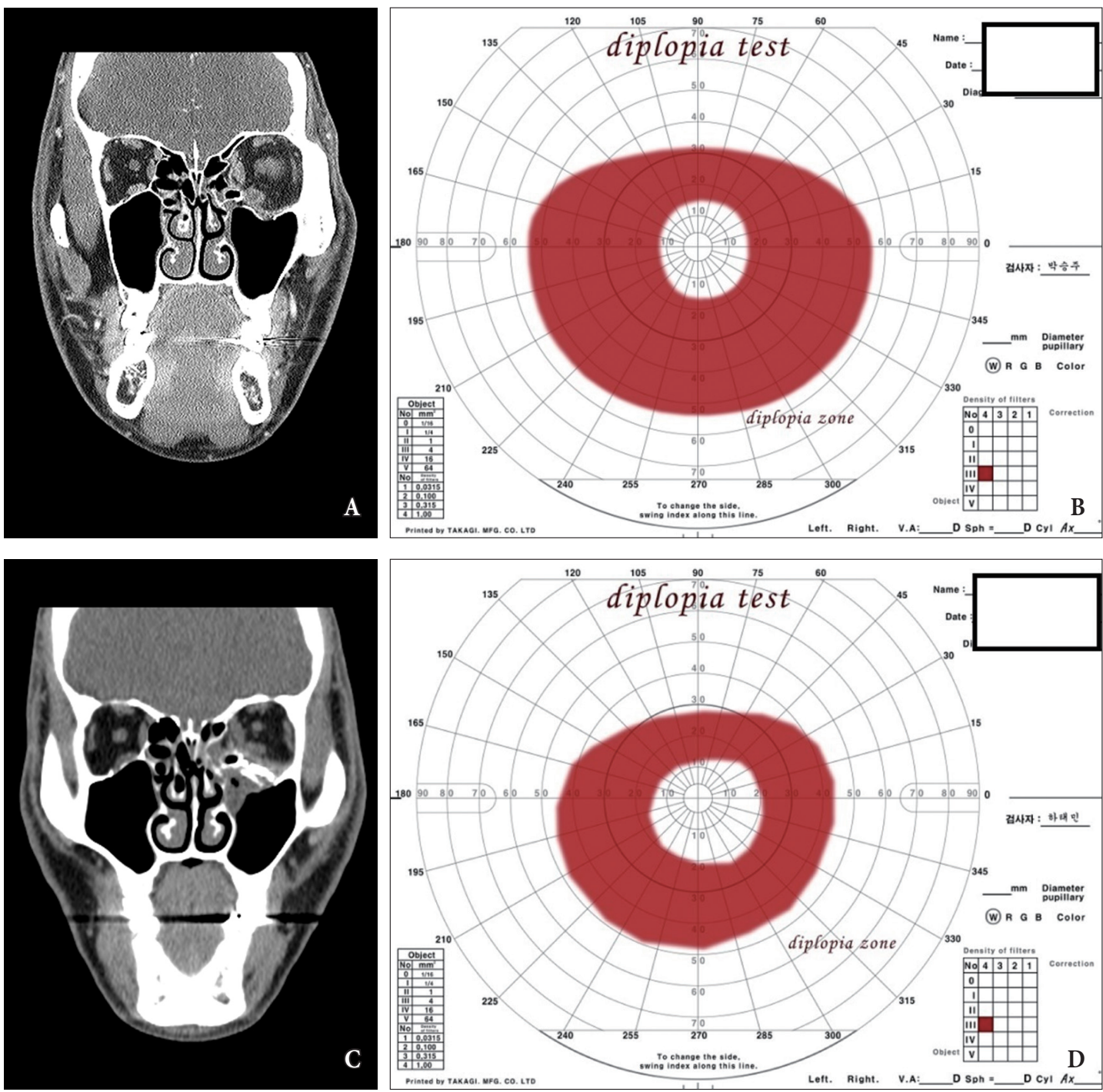

Fig. 7. A patient with persistent diplopia for 11 months following orbital floor reconstruction. (A) At the time of the initial operation, the left medial wall and floor were repaired with an absorbable plate. (B) Diplopia test shows diplopia zone (red zone). Absorbable plates were removed and scar tissue was released in periorbital tissue during the secondary operation. The orbital floor was reconstructed with a titanium plate (C). Diplopia zone was markedly decreased in diplopia test (D).
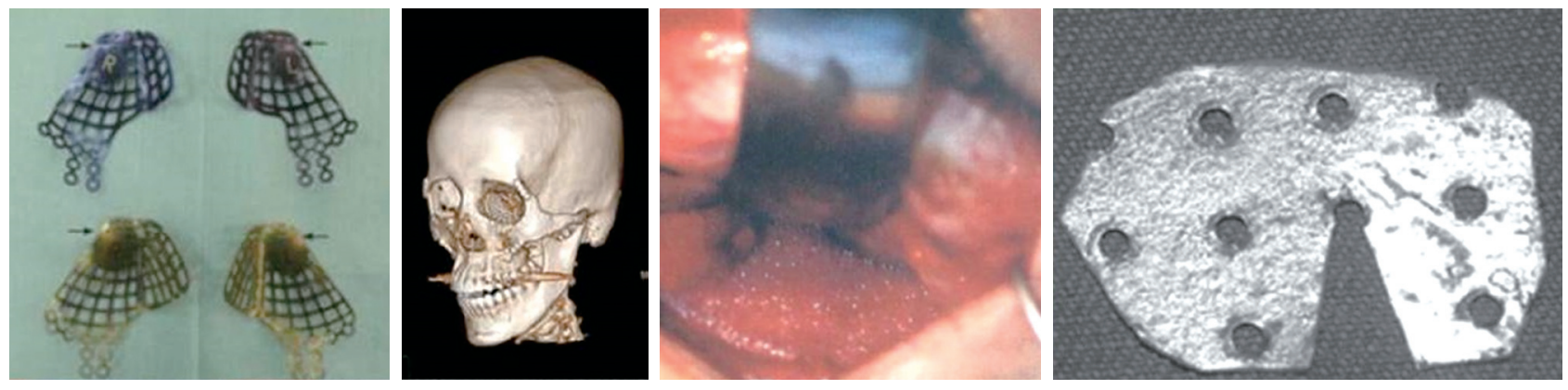

Fig. 8. Various implants available for orbital floor reconstruction. 


\section{REFERENCES}

1. Nakamura T, Gross CW. Facial fractures: analysis of five years of experience. Arch Otolaryngol 1973;97:288-90.

2. Gwyn PP, Carraway JH, Horton CE, Adamson JE, Mladick RA. Facial fractures: associated injuries and complications. Plast Reconstr Surg 1971;47:225-30.

3. Rontal E, Rontal M, Guilford FT. Surgical anatomy of the orbit. Ann Otol Rhinol Laryngol 1979;88:382-6.

4. Rootman J. Basic anatomic considerations. In: Rootman J, editor. Diseases of the orbit : a multidisciplinary approach. Philadelphia: Lippincott; 1988.p. 3-18.

5. Ellis E3rd, el-Attar A, Moos KF. An analysis of 2,067 cases of zygomatico-orbital fracture. J Oral Maxillofac Surg 1985;43:417-28.

6. Smith B, Regan WF Jr. Blow-out fracture of the orbit; mechanism and correction of internal orbital fracture. Am J Ophthalmol 1957;44:733-9.

7. Hawes MJ, Dortzbach RK. Surgery on orbital floor fractures: influence of time of repair and fracture size. Ophthalmology 1983;90:1066-70.

8. Boyette JR, Pemberton JD, Bonilla-Velez J. Management of orbital fractures: challenges and solutions. Clin Ophthalmol 2015;9:2127-37.

9. Chen CT, Huang F, Chen YR. Management of posttraumatic enophthalmos. Chang Gung Med J 2006;29:251-61.

10. Sires BS, Stanley RB Jr, Levine LM. Oculocardiac reflex caused by orbital floor trapdoor fracture: an indication for urgent repair. Arch Ophthalmol 1998;116:955-6.

11. Kang MS, Jeong EC. White-eyed medial orbital wall fracture with oculocardiac symptoms. Arch Plast Surg 2015;42:247-9.

12. Smith B, Lisman RD, Simonton J, Della Rocca R. Volkmann's contracture of the extraocular muscles following blowout fracture. Plast Reconstr Surg 1984;74:200-16.

13. Parsons GS, Mathog RH. Orbital wall and volume relationships. Arch
Otolaryngol Head Neck Surg 1988;114:743-7.

14. Aldekhayel S, Aljaaly H, Fouda-Neel O, Shararah AW, Zaid WS, Gilardino M. Evolving trends in the management of orbital floor fractures. J Craniofac Surg 2014;25:258-61.

15. Dutton JJ. Management of blow-out fractures of the orbital floor. Surv Ophthalmol 1991;35:279-80.

16. Hammer B. Orbital fractures: diagnosis, operative treatment, secondary corrections. Seattle: Hogrefe \& Huber Publishers; 1995.

17. Strong EB, Kim KK, Diaz RC. Endoscopic approach to orbital blowout fracture repair. Otolaryngol Head Neck Surg 2004;131:683-95.

18. Salgarelli AC, Bellini P, Landini B, Multinu A, Consolo U. A comparative study of different approaches in the treatment of orbital trauma: an experience based on 274 cases. Oral Maxillofac Surg 2010;14:23-7.

19. Ord RA, El Attar A. Acute retrobulbar hemorrhage complicating a malar fracture. J Oral Maxillofac Surg 1982;40:234-6.

20. Brucoli M, Arcuri F, Cavenaghi R, Benech A. Analysis of complications after surgical repair of orbital fractures. J Craniofac Surg 2011;22:1387-90.

21. Hosal BM, Beatty RL. Diplopia and enophthalmos after surgical repair of blowout fracture. Orbit 2002;21:27-33.

22. Lee KM, Park JU, Kwon ST, Kim SW, Jeong EC. Three-dimensional pre-bent titanium implant for concomitant orbital floor and medial wall fractures in an East Asian population. Arch Plast Surg 2014;41:480-5.

23. Ng SG, Madill SA, Inkster CF, Maloof AJ, Leatherbarrow B. Medpor porous polyethylene implants in orbital blowout fracture repair. Eye (Lond) 2001;15:578-82.

24. Tuncer S, Yavuzer R, Kandal S, Demir YH, Ozmen S, Latifoglu O, et al. Reconstruction of traumatic orbital floor fractures with resorbable mesh plate. JCraniofac Surg 2007;18:598-605. 